Reprod. Nutr. Develop., 1988, 28 (3 B), 757-768

\title{
Polymorphisme de l'acétylcholinestérase et de la myosine au cours du développement des muscles rapide et lent dénervés chez le lapin nouveau-né
}

\author{
F. BACOU, P. VIGNERON
}

avec la collaboration technique de L. MARGER et Marylène ROSTAGNO

Station de Physiologie animale, I.N.R.A.-E.N.S.A.M. 34060 Montpellier Cedex, France.

Summary. Polymorphism of acety/cholinesterase and myosin during the development of slow and fast muscle fibers after neonatal denervation in the rabbit.

The rabbit Semimembranosus proprius (SMp) and Semimembranosus accessorius (SMa) muscles represent good models for studying the transformations of muscle properties during postnatal differentiation. In the adult, these muscles are homogeneous in slow twitch (SMp) and fast twitch (SMa) fibers, respectively. However, they are heterogeneous at birth and express their adult characteristics from two months onwards. During this period we studied the influence of motor innervation on the development of their properties, particularly at the level of acetylcholinesterase (AChE) molecular forms and myosin slow (LCs) and fast (LCf) light chains.

The postnatal alteration of SMa and SMp muscles was characterized by the disappearance of the neonatal heterogeneity and the acquisition of the homogeneous fast or slow fiber type pattern. The fibers of these muscles denervated at birth were altered differently: dramatic atrophy of fast twitch fibers whatever the muscles studied, preservation of SMp slow twitch fiber characteristics and fatty degeneration of SMa.

At birth, both muscles presented a similar pattern of myosin fast and slow LC. In control muscles, the alteration of fiber populations to homogeneous types led to the disappearance of supernumerary chains from 15 days onwards. In the slow muscle, neonatal denervation prevented LCf disappearance. In the fast muscle, denervation influenced essentially the installation of LCf which was delayed by 15 days.

At birth, the polymorphism of AChE was similar in SMp and SMa muscles. One month after denervation, the specific activity of AChE was twice that of the control. Its polymorphism was not much disturbed, while in the adult denervation induced a large increase in AChE specific activity $(\times 10)$ and particularly a great alteration in its polymorphism according to the fast or slow muscle fiber types.

\section{Introduction.}

De nombreuses expériences ont montré que le type d'activité contractile des muscles est sous l'influence du système nerveux moteur (Buller et al., 1960b; Close, 1972). Au cours du développement, les changements dans la vitesse de 
contraction musculaire interviennent rapidement dès l'installation de l'innervation motrice dans les muscles d'oiseaux (Srihari et Vrbova, 1978) et progressivement après l'innervation des fibres dans les muscles de mammifères (Huizar et al., 1975).

Chez les mammifères, l'activité motrice se développe pendant la période périnatale. A la naissance, les muscles squelettiques lents ou rapides chez l'adulte se contractent encore lentement mais déjà différemment, et augmentent leur vitesse de contraction dans les premières semaines postnatales pour atteindre leurs caractéristiques contractiles adultes lente, rapide et intermédiaire (Buller et al., 1960a ; Close, 1964 ; Drachman et Johnston, 1973).

Les muscles Semimembranosus accessorius (SMa) et Semimembranosus proprius $(\mathrm{SMp})$ de lapin représentent un modèle remarquable d'étude des transformations intervenant dans la différenciation néonatale des propriétés musculaires. Ces muscles homogènes chez l'adulte (Appel et Hammersen, 1979; planche $A$, a et d) respectivement en fibres rapides ou de type II (SMa) et en fibres lentes ou de type I (SMp), sont hétérogènes à la naissance et acquièrent leurs caractéristiques fibrillaires définitives dans les deux mois postnataux.

Nous avons étudié sur ces deux muscles, au cours de cette période critique de leur différenciation, le rôle de l'innervation motrice dans l'établissement et le maintien de leur schéma fibrillaire, en nous intéressant à deux protéines impliquées dans le fonctionnement du tissu musculaire: la myosine, protéine contractile majeure, dont nous avons étudié la répartition des chaînes légères lentes et rapides, et l'acétylcholinestérase (AChE, EC : 3.1.1.7), enzyme polymorphe intervenant dans la dégradation de l'acétylcholine libérée à la synapse, donc dans la régulation des interactions fonctionnelles neuro-musculaires.

\section{Matériel et méthodes.}

Cette série d'expériences a été réalisée sur des lapereaux de race néozélandaise provenant de notre élevage.

Protocole de dénervation néonatale. - Les muscles SMa et SMp sont innervés par un tronc commun issu du nerf sciatique (Kerr, 1955). La dénervation a été effectuée selon le protocole utilisé chez l'adulte (Bacou et al., 1985). Les lapereaux, âgés de 1 jour, ont été anesthésiés au Ketalar $50(50 \mathrm{mg} / \mathrm{ml}$ de kétamine base) injecté par voie intrapéritonéale à raison de $100 \mu \mathrm{l} / 100 \mathrm{~g} \mathrm{de}$ poids.

Etude histochimique. - Les coupes de $12 \mu \mathrm{m}$ d'épaisseur ont été effectuées au cryostat, les muscles étant préalablement congelés dans l'isopentane refroidi à - $70^{\circ} \mathrm{C}$ par de la neige carbonique. Les types de fibres musculaires ont été mis en évidence par la révélation de l'activité ATPasique de l'actomyosine après préincubation alcaline à $\mathrm{pH} 9,4$ (Guth et Samaha, 1969). Les fibres de type rapide peuvent ainsi être déterminées au microscope par leur coloration sombre, les fibres de type lent apparaissant en clair. 
Caractérisation des chaînes légères de la myosine. - Les chaînes légères de la myosine sont séparées sur gel d'électrophorèse à deux dimensions. L'électrofocalisation est réalisée dans des tubes $(100 \mathrm{~mm} \times 1,5 \mathrm{~mm}$ de diamètre interne) de gel polyacrylamide (3,64 \%) selon la technique de O'Farrel (1975) avec $2 \%$ d'un mélange d'ampholines LKB ( $\mathrm{pH} 3,5-10 ; 4-6 ; 5-7)$. La deuxième électrophorèse en présence de sodium dodécyl sulfate (SDS, $0,1 \%$ ) est effectuée sur plaque de gels ( 1 ou $1,5 \mathrm{~mm}$ d'épaisseur) de polyacrylamide $(0,3 \%$ bis) selon Laemmli (1970) avec un gel de concentration de $4,5 \%(3,5 \mathrm{~cm}$ de hauteur $\times 14 \mathrm{~cm}$ de longueur) et un gel de séparation de $15 \%(10 \mathrm{~cm}$ de hauteur $\times 14 \mathrm{~cm}$ de longueur).

Etude du polymorphisme de l'acéty/cholinestérase. - L'acétylcholinestérase a été extraite des tissus musculaires dans un tampon salin à haute force ionique en présence d'antiprotéolytiques $(\mathrm{NaCl} 1 \mathrm{M}$, Triton $X 1001 \%$, Tris $\mathrm{HCl} 10 \mathrm{mM} \mathrm{pH} 7$, benzamidine $1 \mathrm{mM}$ et aprotinine $25 \mathrm{U} / \mathrm{ml}$ ). Les différentes formes moléculaires de l'enzyme ont été caractérisées sur gradient de saccharose, selon la technique classique de Massoulié et Rieger (1969) couramment utilisée au laboratoire (Bacou, 1982).

\section{Résultats.}

1) Evolution postnatale des types de fibres. - A la naissance, les muscles $\mathrm{SMa}$ et SMp sont tous deux hétérogènes, le pourcentage des différents types de fibres évoluant progressivement vers le schéma adulte atteint dès deux mois.

Les deux muscles manifestent une évolution différente après dénervation spécifique pratiquée à la naissance. Le muscle SMa, de type rapide chez l'adulte, montre une atrophie considérable de l'ensemble des fibres qui le constitue, associée à une dégénérescence adipeuse importante (planche $A, b$ et $c$ ). Ainsi, à 30 jours postopératoire, le muscle ne contient que quelques fibres noyées dans une masse adipeuse qui constitue l'ensemble du "muscle» après deux mois d'opération.

Au contraire, la structure du muscle SMp, de type lent chez l'adulte, est beaucoup moins sensible à la dénervation. En particulier, les fibres de type l qui le constituent en majorité persistent, passant par un stade d'hypertrophie après 15 jours postopératoire (planche $A$, e et $f$ ) pour conserver une taille voisine de celle des témoins après un mois de dénervation. Seules, les fibres de type II, qui cohabitent avec les précédentes au cours de cette période, s'atrophient considérablement (planche $A, c$ et $f$ ), n'existant que sous forme de reliquat après un mois d'opération.

2) Evolution des chaînes légères de la myosine (tabl. 1). - Outre des caractéristiques différentes au niveau de leur chaîne lourde, chaque type de myosine présente une composition particulière en sous-unités de faible poids moléculaire ou chaînes légères (LC) qui peuvent servir de marqueur conventionnel des propriétés contractiles des muscles (Gauthier et al., 1982; Lowey and Risby, 1971). On distingue ainsi chez le lapin, associés aux lourdes chaînes 


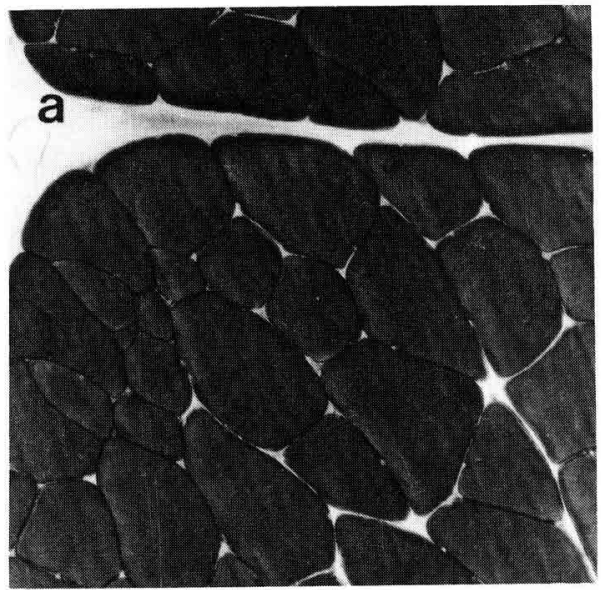

350

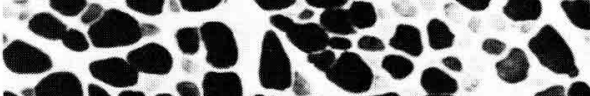
2003. Lus - 1 r. atis.

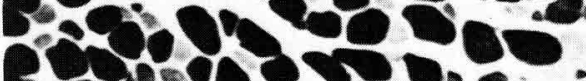
tres 30. Coses:

\% ran (5) C.;

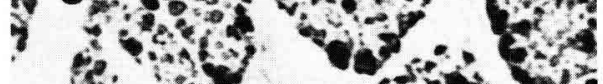

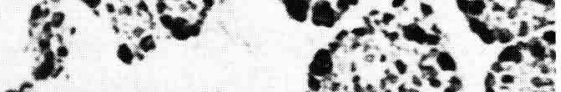

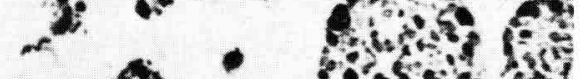
- s.

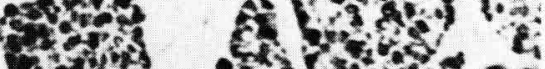

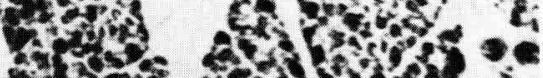

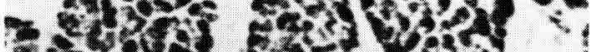

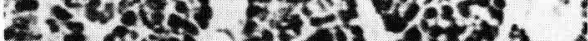

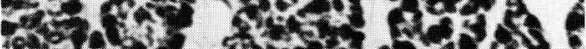
iscos $x$ 2 of 1 (s) atmentisens.

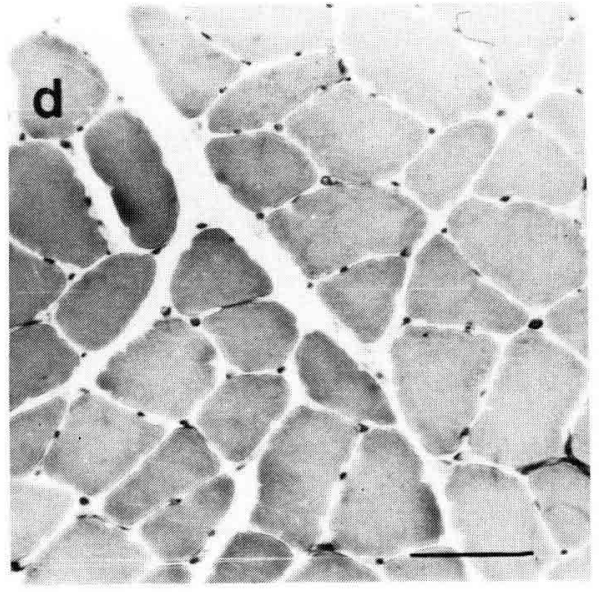

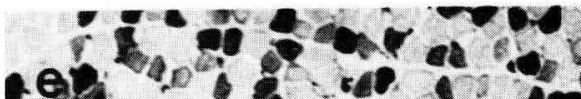

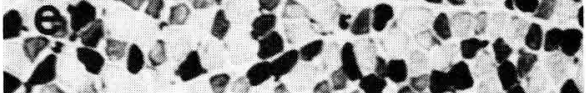
$\rightarrow$ - कर ke

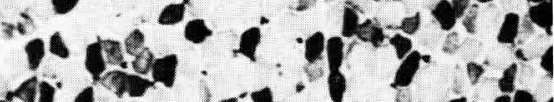

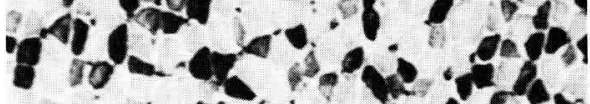

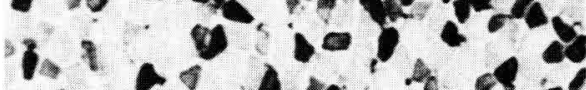

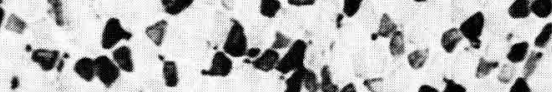

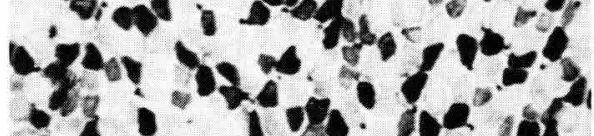

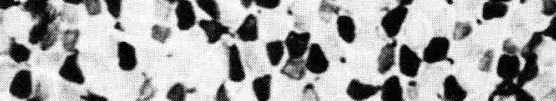
, 16400,5

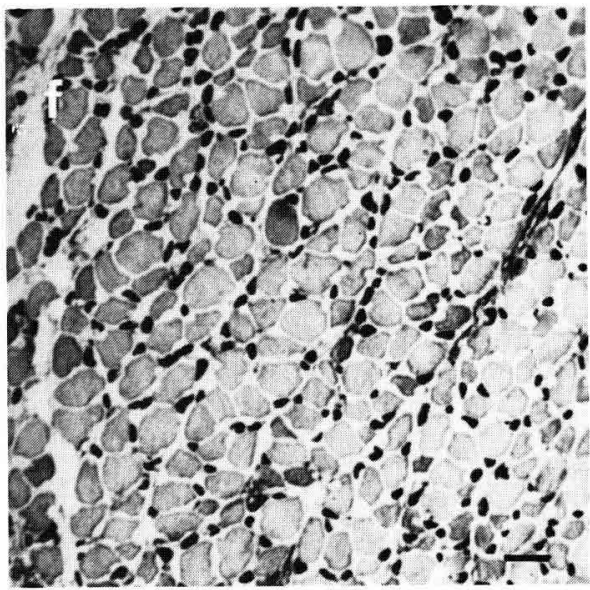


polypeptidiques, trois types de chaînes légères rapides: $L C_{1} f, L C_{2} f$ et $L C_{3} f$, et des chaînes légères lentes: $L_{1} \mathrm{~S}_{1}, \mathrm{LC}^{\prime}{ }_{1} \mathrm{~s}$ et $\mathrm{LC}_{2} \mathrm{~s}$. $\mathrm{A}$ ces catégories de chaînes légères caractéristiques des muscles adultes, on trouve au cours de la période prénatale une chaîne légère embryonnaire: $L_{1}$ emb. (Whalen et al., 1978).

Chez l'adulte, les muscles SMa et SMp ont une constitution en LC différente, respectivement $L_{1} \mathrm{f}_{1}, \mathrm{LC}_{2} \mathrm{f}, \mathrm{LC}_{2} \mathrm{~S}(\mathrm{SMa})$ et $\mathrm{LC}_{1} \mathrm{~s}, \mathrm{LC}_{1}{ }_{1} \mathrm{~s}, \mathrm{LC}_{2} \mathrm{~S}(\mathrm{SMp})$. A la naissance, au contraire, ces deux tissus présentent un assortiment complet des chaînes légères lentes et rapides de la myosine adulte. Chez l'animal normal, l'évolution au cours de la croissance des populations de fibre vers des types homogènes conduit à la disparition des chaînes surnuméraires.

a) Muscle Semimembranosus accessorius. - L'évolution des LC de la myosine au cours de la période néonatale est caractérisée par l'augmentation progressive des $L C_{f}$, leurs pourcentages respectifs étant pratiquement stabilisés dès un mois. Par ailleurs, les chaînes $\mathrm{LC}_{1} \mathrm{~s}$ et $\mathrm{LC}_{1}{ }_{1} \mathrm{~s}$ disparaissent à des stades variables mais à un mois postnatal, le polymorphisme en LC de la myosine est semblable à celui observé chez l'adulte.

La dénervation altère sensiblement l'évolution de ces caractéristiques. L'installation des LCf est retardée, en particulier dans les 15 premiers jours postnataux. De plus, on observe le maintien de $\mathrm{LC}_{1} \mathrm{~s}$ à tous les stades étudiés. La dégénérescence adipeuse caractérisant ce type de muscle dénervé ne nous permet pas de pousser nos investigations au-delà de un mois postopératoire.

b) Muscle Semimembranosus proprius. - A la naissance, outre l'ensemble des LC de myosine adulte, on détecte dans le muscle lent la forme LC $_{1}$ embryonnaire. Par la suite, cette forme disparaît en quelques jours, tandis que la quantité des LCs augmente rapidement. Parallèlement, les LCf diminuent et ont pratiquement disparu à un mois. Comme dans le muscle rapide, le polymorphisme en LC de la myosine du muscle lent est semblable à celui de l'adulte dès le premier mois de vie postnatale.

Le maintien de la structure musculaire après dénervation nous a permis d'étudier à plus long terme l'évolution des LC du muscle SMp opéré. Elles est caractérisée par a) le retard dans la disparition des $\mathrm{LC}_{1}$ emb., présentes en quantité notable à 15 jours ; - b) le retard dans la mise en place des LCs ; - c) le maintien quel que soit le stade étudié des trois LCf. Le muscle de SMp dénervé adulte est donc caractérisé par l'ensemble des chaînes lentes et rapides, seule la forme embryonnaire ayant tardivement disparu.

\section{PLANCHE A}

Caractérisation par l'ATPase myofibrillaire après incubation a/caline des fibres de type / (claires) et de type // (foncées). a) Muscle Semimembranosus accessorius adulte ; b) Muscle Semimembranosus accessorius de 15 jours ; $c$ ) Muscle Semimembranosus accessorius 15 jours après dénervation à la naissance ; d) Muscle Semimembranosus proprius adulte ; e) Muscle Semimembranosus proprius de 15 jours; f) Muscle Semimembranosus proprius 15 jours après dénervation à la naissance.

a et $\mathrm{d}$ même échelle $=100 \mu \mathrm{m}(\mathrm{d})$

b, $c$, e et f même échelle $=100 \mu \mathrm{m}(\mathrm{f})$. 


\begin{tabular}{|c|c|c|c|c|c|}
\hline Myosine & Naiss. & $6 \mathrm{j}$ & $15 j$ & 1 mois & Adulte \\
\hline LC $1 \mathrm{emb}$. & + & $\begin{array}{c}0 \\
++\end{array}$ & $\begin{array}{l}0 \\
+\end{array}$ & $\begin{array}{l}0 \\
0\end{array}$ & $\begin{array}{l}0 \\
0\end{array}$ \\
\hline LC'1s & ++ & $\begin{array}{c}++t \\
++\end{array}$ & $\begin{array}{c}++++ \\
+++\end{array}$ & $\begin{array}{c}++++ \\
+++\end{array}$ & $\begin{array}{l}++++ \\
++++\end{array}$ \\
\hline LC T's & ++ & $\begin{array}{c}+++ \\
++\end{array}$ & $\begin{array}{c}++++ \\
+++\end{array}$ & $\begin{array}{l}++++ \\
++++ \\
\end{array}$ & $\begin{array}{c}++++ \\
+++ \\
\end{array}$ \\
\hline LC 25 & ++ & $\begin{array}{c}++ \\
++\end{array}$ & $\begin{array}{c}++++ \\
+++\end{array}$ & $\begin{array}{l}++++ \\
++++\end{array}$ & $\begin{array}{l}++++ \\
++++\end{array}$ \\
\hline LCIf & ++ & $\begin{array}{l}++ \\
++\end{array}$ & $\begin{array}{c}+ \\
++\end{array}$ & $\begin{array}{l}+10 \\
++ \\
\end{array}$ & $\begin{array}{c}0 \\
+++\end{array}$ \\
\hline $\operatorname{LC} 2 f$ & + & $\begin{array}{l}0 \\
+ \\
\end{array}$ & $\begin{array}{c}0 \\
+10 \\
\end{array}$ & $\begin{array}{r}0 \\
+10 \\
\end{array}$ & $\begin{array}{r}0 \\
+10 \\
\end{array}$ \\
\hline $\operatorname{LC} 3 \mathrm{f}$ & $\begin{array}{c}+10 \\
1 \\
\end{array}$ & $\begin{array}{c}+10 \\
+ \\
\end{array}$ & $\begin{array}{l}0 \\
+ \\
\end{array}$ & $\begin{array}{r}0 \\
+ \\
\end{array}$ & $\begin{array}{l}0 \\
+ \\
\end{array}$ \\
\hline
\end{tabular}

\begin{tabular}{|c|c|c|c|c|c|}
\hline Myosine & Naiss. & $6 j$ & $15 \mathrm{j}$ & 1 mois & Adulte \\
\hline LC 1 emb & 0 & $\begin{array}{l}0 \\
0\end{array}$ & $\begin{array}{l}0 \\
0\end{array}$ & $\begin{array}{l}0 \\
0\end{array}$ & 0 \\
\hline LC'15 & + & $\begin{array}{l}0 \\
0\end{array}$ & $\begin{array}{l}+ \\
0\end{array}$ & $\begin{array}{l}0 \\
0\end{array}$ & 0 \\
\hline LC 15 & + & $\begin{array}{l}+ \\
+\end{array}$ & $\begin{array}{l}+ \\
+\end{array}$ & $\begin{array}{l}0 \\
+\end{array}$ & ; \\
\hline LC 25 & ++ & $\begin{array}{l}++ \\
++ \\
\end{array}$ & $\begin{array}{l}++ \\
++\end{array}$ & $\begin{array}{l}++ \\
++ \\
\end{array}$ & $\begin{array}{c}+++ \\
\end{array}$ \\
\hline LC1f & + & $\begin{array}{c}+++ \\
++\end{array}$ & $\begin{array}{c}++++ \\
+++\end{array}$ & $\begin{array}{l}++++ \\
++++\end{array}$ & $\begin{array}{c}+++ \\
\quad\end{array}$ \\
\hline LC $2 f$ & + & $\begin{array}{l}+ \\
+\end{array}$ & $\begin{array}{c}+ \\
++ \\
\end{array}$ & $\begin{array}{l}++ \\
++ \\
\end{array}$ & + \\
\hline LC $3 f$ & + & $\begin{array}{c}++ \\
+\end{array}$ & $\begin{array}{c}+++ \\
+\end{array}$ & $\begin{array}{c}+++ \\
++\end{array}$ & $\begin{array}{c}++++ \\
\end{array}$ \\
\hline
\end{tabular}

\section{TABLEAU 1}

Evolution au cours de la vie postnatale des chaînes légères de la myosine (voir texte) dans les muscles (A) Semimembranosus proprius et (B) Semimembranosus accessorius normaux ou dénervés à la naissance. Dans chacune des colonnes, les valeurs de la ligne supérieure correspondent aux muscles témoins, celles de la ligne inférieure aux muscles dénervés.

3) Evolution du polymorphisme de l'acétylcholinestérase (fig. 1). - L'AChE est une enzyme polymorphe ubiquitaire, essentiellement localisée dans les muscles au niveau de la jonction neuromusculaire (McMahan et al., 1978). L'AChE existe dans les tissus musculaires des vertébrés selon deux types de formes: globulaires et asymétriques (Massoulié et Bon, 1982). 

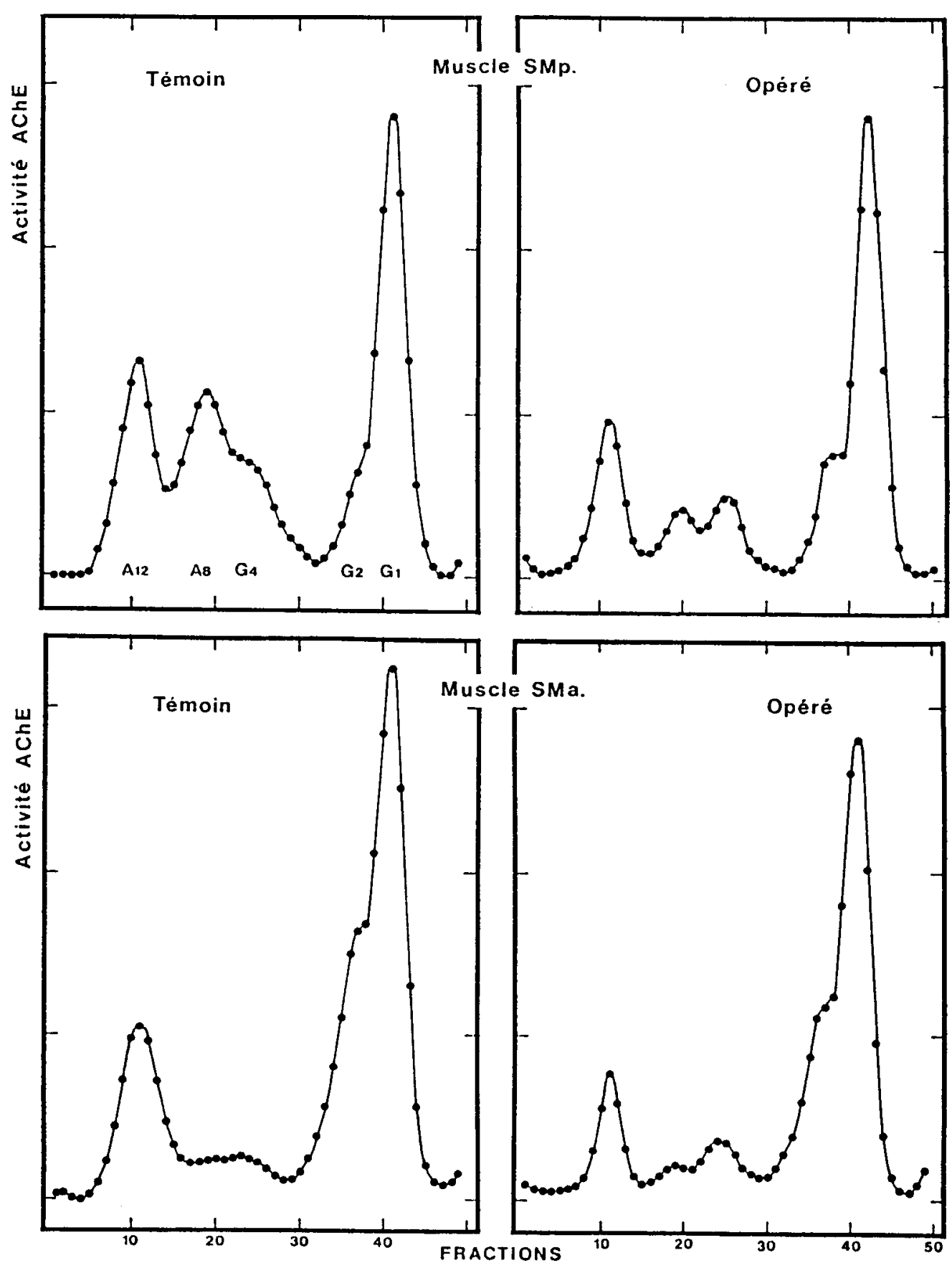

FIG. 1. - Polymorphisme de l'acétylcholinestérase des muscles Semimembranosus proprius (SMp) et Semimembranosus accessorius (SMa) témoins ou après 1 mois de dénervation à la naissance. 
Nous avons étudié chez le lapereau la régulation de la synthèse de cette enzyme et de ses formes moléculaires à un âge ou l'innervation motrice et les types de fibres musculaires n'ont pas atteint leurs caractéristiques adultes. Le polymorphisme de l'AChE à la naissance présente dans les deux muscles un schéma analogue caractérisé par l'importance des formes asymétriques qui constituent 40 à $50 \%$ de l'activité acétylcholinestérasique totale. Au cours du développement, le polymorphisme évolue dans les deux muscles avec, en particulier, une diminution importante des formes asymétriques. Après dénervation des muscles à la naissance, l'activité spécifique de l'enzyme n'est accrue que d'un facteur deux à un mois postopératoire. De même, le polymorphisme n'est que légèrement perturbé, et on observe dans les stades étudiés et jusqu'à un mois postdénervation un pourcentage de formes asymétriques voisin des témoins quel que soit le type de muscle.

\section{Discussion.}

Comme cela a déjà été montré sur d'autres espèces de mammifères, les muscles homogènes chez l'adulte, hétérogènes à la naissance, n'acquièrent que progressivement leurs caractéristiques définitives (Karpati et Engel, 1968 ; Okada et al., 1984), cette évolution intervenant dans les deux mois postnataux chez le lapin. Le déterminisme qui gouverne la différenciation fonctionnelle lente ou rapide des fibres musculaires n'agit donc effectivement qu'après la naissance. Parmi les facteurs déterminant cette différenciation et comme le montrent nos expériences de dénervation néonatale, l'innervation motrice jouerait un rôle essentiel : d'une part dans le maintien de la structure musculaire (voir par exemple la dégénérescence adipeuse rapide du muscle SMa dénervé), d'autre part dans l'établissement du schéma en type de fibres adultes. Ce dernier rôle de l'innervation dans la période néonatale pourrait découler de la suppression de la polyinnervation des fibres musculaires (Bennett et al., 1973 ; Bennett et Pettigrew, 1974; Brown et al., 1976), ou d'une modification postnatale des caractéristiques nerveuses motrices (Huizar et al., 1975), les deux hypothèses n'étant pas mutuellement exclusives. En particulier, l'activité motrice imposée aux muscles par leur innervation spécifique semble avoir un rôle prépondérant (Salmons et Vrbova, 1969 ; Salmons et Sreter, 1976), qui pourrait expliquer en partie l'atrophie et la dégénérescence des muscles étudiés.

Comme nous l'avons observé au niveau de la myosine, la dénervation empêche la ségrégation des LC caractéristiques des tissus différenciés. II a déjà été établi par dénervation (Gauthier et Hobbs, 1982 ; Carraro et al., 1985) ou innervation croisée (Sreter et al., 1985) ou innervation croisée (Sreter et al., 1974, 1975 ; Gauthier et al., 1983) chez l'adulte que l'innervation motrice est indispensable à la stabilité des caractéristiques de la myosine. L'acquisition et le maintien des types de myosine chez le jeune et chez l'adulte sont donc étroitement liés à la présence de l'innervation motrice. L'installation d'une innervation motrice fonctionnelle induirait la différenciation des divers types de fibre en éliminant en particulier les chaînes embryonnaires existant dans la période prénatale, et en favorisant l'expression des myosines soit lentes, soit rapides dans un type de muscle donné (Brown et al., 1983). 
Contrairement à ce qui est observé pour la myosine, la comparaison de la distribution des différentes formes de l'AChE des muscles dénervés chez le lapin adulte et le nouveau-né montre que l'innervation motrice exerce à ces deux stades une influence différente. L'étude des formes asymétriques présente un intérêt particulier car elles apparaissent dès l'installation de l'innervation motrice au cours de l'embryogenèse (Kato et al., 1980 ; Vigny et al., 1976b). Disparaissant après dénervation chez le rat (Collins et Younkin, 1982 ; Hall, 1973) et le poulet (Sketelj et al., 1978 ; Vigny et al., 1976a), elles ont été longtemps considérées comme un marqueur biochimique de jonctions neuromusculaires fonctionnelles. Chez le lapin cependant, nous avons montré que ce comportement dépendait du type de muscle étudié (Bacou et al., 1982). Après dénervation des muscles SMa et SMp de lapin adulte, nous avons constaté deux phénomènes : a) l'activité spécifique de l'AChE est multipliée par un facteur dix, quel que soit le type de muscle; $b$ ) un profond remaniement de son polymorphisme intervient, en relation avec le type rapide ou lent des fibres musculaires. Le polymorphisme de l'AChE de muscle SMa adulte dénervé présente le schéma classique caractérisé par la disparition des formes asymétriques. Dans le muscle SMp dénervé, au contraire, les formes asymétriques sont néosynthétisées et la présence de la forme $A_{12}$, la plus lourde, est accrue d'un facteur vingt. Chez l'adulte, la synthèse de formes asymétriques est donc étroitement corrélée aux types de fibres. En particulier, les résultats obtenus sur le muscle lent dénervé - néosynthèse des formes asymétriques - et confirmés depuis sur d'autres muscles de lapin et de cobaye (Lai et al., 1986), remettent en cause les idées dominantes depuis plus de dix ans sur le rôle indispensable de l'innervation motrice dans la synthèse de ces formes moléculaires. Au cours de la période périnatale, les fibres musculaires présentent une indépendance relative quel que soit leur type et synthétisent en présence et en absence d'innervation motrice toutes les formes d'AChE. Ces résultats sont à rapprocher de ceux obtenus in vitro sur des myotubes différenciés à partir de myoblastes prélevés chez des embryons de rat (Koenig et al., 1984) et de poulet (Kato et al., 1980): les formes asymétriques de l'AChE sont synthétisées indépendamment de l'activité motrice per se (de La Porte et al., 1984) dans les cultures aneurales de muscles, la régulation de la synthèse de cette enzyme étant probablement liée à la perméabilité ionique de la fibre musculaire.

Le comportement de ces muscles incomplètement différenciés pose la question des interactions nerf/muscle dans la période néonatale. L'évolution des relations neuromusculaires suppose des plaques motrices fonctionnelles puisque c'est à leur niveau que la transmission nerf/muscle s'effectue. Les données obtenues avec l'AChE montrent qu'au cours de la vie néonatale, les constituants de la jonction neuromusculaire n'ont pas les mêmes propriétés relatives à l'innervation motrice que chez l'adulte, et l'ensemble de nos résultats atteste la nécessité d'une maturation préalable des caractéristiques des relations neuromusculaires, cette maturation intervenant progressivement au cours des premiers mois postnataux.

En conclusion, le déterminisme nerveux de la différenciation musculaire s'effectue différemment selon les critères considérés. Sur le plan strictement musculaire, l'innervation motrice est dès la naissance nécessaire à l'établissement

Reproduction, Nutrition, Développement, $n^{\circ} 3$ B. -7 
des caractéristiques adultes. Nous avons vu l'effet important de la dénervation sur les propriétés histochimiques des fibres musculaires, en particulier les différences de sensibilité entre fibres lentes - qui demeurent dans le muscle lent - et des fibres rapides qui subissent une atrophie considérable dans les deux types de muscle. Au niveau de la myosine, les chaînes légères - et les chaînes lourdes sont de type lent ou rapide, et dépendent intimement du type d'innervation et du type d'activité motrice associée.

Au contraire, certains constituants de la jonction neuromusculaire, comme l'AChE, ne sont pas aussi étroitement liés à l'innervation motrice dans la période néonatale. Les plaques motrices, structures mixtes neuromusculaires, reçoivent chez l'adulte à la fois des informations stabilisées d'origine nerveuse et d'origine musculaire. On peut penser qu'au cours de leur maturation dans la période néonatale, un état d'équilibre réciproque s'établit progressivement entre les deux tissus afin que cette structure puisse exprimer et stabiliser les propriétés que nous avons observés chez l'adulte.

$13^{e}$ Réunion du groupe Développement I.N.R.A. Cap d'Adge, 25-27 mai 1987.

\section{Références}

APPEL H. J., HAMMERSEN F., 1979. The fiber composition of the semitendinosus muscle of the rabbit. Cell Tissue Res., 196, 531-539.

BACOU F., 1982. The polymorphism of acetylcholinesterase in different animal species and motor innervation. Reprod. Nutr. Dévelop., 22, 227-233.

BACOU F., VIGNERON P., MASSOULIE J., 1982. Acetylcholinesterase forms in fast and slow rabbit muscle. Nature, 296, 661-664.

BACOU F., VIGNERON P., COURAUD J. Y., 1985. Retrograde effect of muscle on forms of acetylcholinesterase in peripheral nerves. J. Neurochem., 45, 1178-1185.

BENNETT M. R., PETTIGREW A. G., TAYLOR R. S., 1973. The formation of synapses in reinnervated and cross-reinnervated adult avian muscle. J. Physiol., 230, 331-357.

BENNETT M. R., PETTIGREW A. G., 1974. The formation of synapses in striated muscle during development. J. Physiol., 241, 515-545.

BROWN M. C., JANSEN J. K. S., VAN ESSEN D., 1976. Polyneuronal innervation of skeletal muscle in new-born rats and its elimination during maturation. J. Physiol., 261, 387-422.

BROWN W. E., SALMONS S., WHALEN R. G., 1983. The sequential replacement of myosin subunit isoforms during muscle type transformation induced by long term electrical stimulation. $J$. biol. Chem., 258, 14686-14692.

BULLER A. J., ECCLES J. C., ECCLES R. M., 1960a. Differentiation of fast and slow muscles in the cat hind limb. J. Physiol., 150, 399-416.

BULLER A. J., ECCLES J. C., ECCLES R. M., 1960b. Interactions between motoneurones and muscles in respect of the characteristic speeds of their responses. J. Physiol., 150, 417-439.

CARRARO U., MORALE D., MUSSINI I., LUCKE S., CANTINI M., BETTO R., CATANI C., DALLA LIBERA L., DANIELI BETTO D., NOVENTA D., 1985. Chronic denervation of rat hemidiaphragm : maintenance of fiber heterogeneity with associated increasing uniformity of myosin isoform. J. Cell Biol., 100, $161-174$.

CLOSE R., 1964. Dynamic properties of fast and slow skeletal muscles of the rat during development. J. Physiol., 173, 74-95.

CLOSE R., 1972. Dynamic properties of mammalian skeletal muscles. Physiol. Rev., 52, 129-197 
COLLINS P. L., YOUNKIN S. G., 1982. Effect of denervation on the molecular forms of acetylcholinesterase in rat diaphragm. J. biol. Chem., 257, 13638-13644.

DE LA PORTE S., VIGNY M., MASSOULIE J., KOENIG J., 1984. Action of Veratridine on acetylcholinesterase in cultures of rat muscle cells. Dev. Biol., 106, 450-456.

DRACHMAN D. B., JOHNSTON D. M., 1973. Development of a mammalian fast muscle : dynamic and biochemical properties correlated. J. Physiol., 234, 29-42.

GAUTHIER G. F., HOBBS A. W., 1982. Effects of denervation on the distribution of myosin isoenzymes in skeletal muscle fibers. Exp. Neurol., 76, 331-346.

GAUTHIER G. F., LOWEY S., BENFIELD P. A., HOBBS A. W., 1982. Distribution and properties of myosin isozymes in developing avian and mammalian skeletal muscle fibers. J. Cell Biol., 92. 471-484.

GAUTHIER G. F., BURKE R. E., LOWEY S., HOBBS A. W., 1983. Myosin isozymes in normal and cross-reinnervated cat skeletal muscle fibers. J. Cell Biol., 97, 756-771

GUTH L., SAMAHA F. J., 1969. Qualitative differences between actomyosin ATPase of slow and fast mammalian muscle. Exp. Neurol., 25, 138-152.

HALL Z. W., 1973. Multiple forms of acetylcholinesterase and their distribution in endplate and non endplate regions of rat diaphragm muscle. J. Neurol., 4, 343-361

HUIZAR P., KUNO M., MIYATA Y., 1975. Differentiation of motoneurones and skeletal muscles in kittens. J. Physiol., 252, 465-479.

KARPATI G., ENGEL W. K., 1968. Correlative histochemical study of skeletal muscle after suprasegmental denervation, peripheral nerve section, and skeletal fixation. Neurology, 18, $681-692$.

KATO A. C., VRACHLIOTIS A., FULPIUS B., DUNANT Y., 1980. Molecular forms of acetylcholinesterase in chick muscle and ciliary ganglion embryonic tissues and cultured cells. Dev. Biol., 76, 222-228.

KERR N. S., 1955. The homologies and nomenclature of the thigh muscles of the opposum, cat, rabbit and rhesus monkey. Anat. Rec., 121, $481-493$.

KOENIG J., DE LA PORTE S., MASSOULIE J., VIGNY M., 1984. Regulation of the acetylcholinesterase molecular forms in nerve-muscle cell cultures. In: G.SERRATRICE et al., Neuromuscular diseases. Raven Press, New York.

LAEMMLI U. K., 1970. Cleavage of structural proteins during the assembly of the head of bacteriophage $T_{4}$. Nature, 227, 680-685.

LAI J., JEDRZEJCZYK J., PIZZEY J. A., GREEN D., BARNARD E. A., 1986. Neural control of the forms of acetylcholinesterase in slow mammalian muscles. Nature, 321, 72-74.

LOWEY S., RISBY D., 1971. Light chains from fast and slow muscle myosins. Nature, 234, $81-85$.

MASSOULIE J., RIEGER F., 1969. L'acétylcholinestérase des organes électriques de Poissons (torpille et gymnote) ; complexes membranaires. Eur. J. Biochem., 11, $441-455$.

MASSOULIE J., BON S., 1982. The molecular forms of cholinesterase and acetylcholinesterase in vertebrates. Ann. Rev. Neurosci., 5, 57-106.

McMAHAN U. J., SANES J. R., MARSHALL L. M., 1978. Cholinesterase is associated with the basal lamina at the neuromuscular junction. Nature, 271, 172-174.

O'FARREL P. H., 1975. High resolution two-dimensional electrophoresis of proteins. J. biol. Chem., 250, 4007-4021.

OKADA S., NOKANA I., CHOU S. M., 1984. Muscle fiber type differentiation and satellite cell populations in normally grown and neonatally denervated muscles in the rat. Acta neuropathol., 65, 90-98.

SALMONS S., VRBOVA G., 1969. The influence of activity on some contractile characteristics of mammalian fast and slow muscles. J. Physiol., 201, 535-549.

SALMONS S., SRETER F. A., 1976. Significance of impulse activity in the transformation of skeletal muscle type. Nature, 263, 30-34.

SKETELJ J., MCNAMEE M. G., WILSON B. W., 1978. Effect of denervation on the molecular forms of acetylcholinesterase in normal and dystrophic chicken muscles. Exp. Neurol., 60, 624-629. 
SRETER F. A., GERGELY J., LUFF A. L., 1974. The effect of cross-reinnervation on the synthesis of myosin light chains. Biochem. biophys. Res. Commun., 56, 84-89.

SRETER F. A., LUFF A. R., GERGELY J., 1975. Effects of cross-reinnervation on physiological parameters and on properties of myosin and sarcoplasmic reticulum of fast and slow muscles of the rabbit. J. gen. Physiol., 66, 811-821.

SRIHARI T., VRBOVA G., 1978. The role of muscle activity in the differentiation of neuromuscular junctions in slow and fast chick muscles. J. Neurocytol., 7, 529-540.

VIGNY M., DI GIAMBERARDINO L., COURAUD J. Y., RIEGER F., KOENIG J., 1976a. Molecular forms of chicken acetylcholinesterase : effect of denervation. FEBS Lett., 69, 277-280.

VIGNY M., KOENIG J., RIEGER F., 1976b. The motor endplate specific form of acetylcholinesterase : appearance during embryogenesis and reinnervation of rat muscle. J. Neurochem., 27. 1347-1353.

WHALEN R. G., BUTLER-BROWNE G. S., GROS F., 1978. Identification of a novel form of myosin light chain present in embryonic muscle tissue and cultured muscle cells. J. mol. Biol., 126, 415-431. 